\title{
Acute effects of dietary fat composition on postprandial plasma bile acid and cholecystokinin concentrations in healthy premenopausal women
}

\author{
Vassiliki Costarelli ${ }^{1,2 *}$ and T. A. B. Sanders ${ }^{2}$ \\ ${ }^{1}$ School of Applied Science, South Bank University, 103 Borough Road, London SE1 OAA, UK \\ ${ }^{2}$ Nutrition, Food and Health Research Centre, King's College London, Franklin-Wilkins Building, 150 Stamford Street, \\ London SE1 9NN, UK
}

(Received 22 December 2000 - Revised 11 April 2001 - Accepted 27 May 2001)

\begin{abstract}
Bile acids derived from intestinal bacterial metabolism and transported to the breast in plasma may influence risk of breast cancer. The purpose of the present study was to test the hypothesis that fatty acid chain length and degree of unsaturation differ with regard to their influence on the postprandial release of cholecystokinin $(\mathrm{CCK})$ and the subsequent increase in plasma bile acid concentrations that occur following a meal. A randomized crossover design was used to compare five high-fat test meals (50 g fat) with a low-fat test meal (15 g) on plasma bile acid and CCK concentrations in eighteen healthy premenopausal women. The high-fat meals were enriched in oleate or palmitate, or linoleate or medium-chain triacylglycerols (MCT) or a blend of oleate and long-chain $n-3$ fatty acids. The postprandial increase in plasma CCK concentration was lower on the MCT meal compared with all meals and was greater following the linoleate compared with the low-fat meal. Plasma bile acid concentrations increased 2-3-fold postprandially but the increase was lower following the MCT meal compared with the other meals and was greater on the linoleate meal compared with the low-fat meal. The postprandial increases in plasma chenodeoxycholic acid concentration showed a trend to rise with increasing unsaturation of the test meal. In conclusion, meals rich in linoleate are a potent stimulus for CCK release and lead to prolonged elevations of plasma bile acids and meals containing MCT inhibit CCK release and the subsequent increase in plasma bile acid concentrations.
\end{abstract}

Bile acids: Cholecystokinin: Dietary fat: Medium-chain triacylglycerol

Hill et al. (1971) proposed that the bile acids deoxycholic acid (DCA) and lithocholic acid which are derived from the bacterial degradation of the primary bile acids, cholic acid (CA) and chenodeoxycholic acid (CDCA) respectively, might be involved in the aetiology of breast cancer. Subsequent studies found fifty-fold higher concentration of DCA and lithocholic acid in human breast cyst fluid than in plasma (Raju et al. 1990; Javitt et al. 1994), indicating that these bile acids accumulated from plasma into breast cysts. DCA is the major secondary bile acid in plasma and lithocholic acid, which is poorly absorbed from the gut, is present in much lower concentrations predominantly in the sulfated form (Bayerdorffer et al. 1995). DCA has been shown to be mutagenic (Watabe \& Bernstein, 1985), to have co-carcinogenic activity (Kawasumi et al. 1988) and to promote the growth and steroid receptor function of MCF-7 human breast cancer cells (Baker et al. 1992). Plasma bile acids may also, in view of their amphipathic properties, enhance the uptake of lipid soluble components such as dioxins, polychlorinated biphenyls and steroids into breast tissue. Plasma bile acid concentrations are low in the fasting state but increase markedly postprandially in response to the action of cholecystokinin (CCK).

CCK secretion is stimulated by the intake of fat, protein and cholesterol (Mössner et al. 1992). Beardshall et al. (1989), in a study of six subjects which investigated the CCK response to meals rich in suet, olive oil and maize oil, reported that the integrated $\mathrm{CCK}$ response to a meal increased according to degree of unsaturation. They concluded that polyunsaturated fats were more potent stimulants of CCK release than saturated fats in man and that the promotion of pancreatic carcinogenesis in rats by unsaturated fatty acids may be related to this effect.

\footnotetext{
Abbreviations: AUC, area under the curve; CA, cholic acid; CCK, cholecystokinin; CDCA, chenodeoxycholic acid; DCA, deoxycholic acid; MCT, mediumchain triacylglycerol.

*Corresponding author: Dr Vassiliki Costarelli, fax +44 207815 7934, email costarv@sbu.ac.uk
} 
However, few other controlled studies have examined the relationship between different dietary fat intake and postprandial levels of plasma bile acids and CCK. Studies on rats which compared maize oil, beef tallow, fish oil and medium-chain triacylglycerols (MCT) concluded that MCT were the most powerful stimulators of CCK secretion (Douglas et al. 1990). The purpose of the present study was to test the hypothesis that fatty acids differ with regard to their effects on the postprandial release of CCK and the subsequent increase in plasma bile acid concentrations in healthy premenopausal women.

\section{Subjects, materials and methods}

\section{Subjects}

Healthy premenopausal female subjects ( $n$ 18) were recruited from the staff and student population of King's College London. A fasting venous blood sample was obtained on screening for haematology, blood lipids and liver function tests, which were determined in the Department of Chemical Pathology, St Thomas's Hospital, London. All subjects were normolipidaemic, non-anaemic and had normal liver function, an intact gastrointestinal tract and gall bladder. Subjects with a BMI $>30 \mathrm{~kg} / \mathrm{m}^{2}$ were excluded, because obesity interferes with gall bladder motility (Kucio et al. 1988). Pregnant and lactating women were excluded and a pregnancy test (Clearview HCGII Pregnancy Test; Wampole Laboratories, PO Box 1001, NJ, USA) was carried out. Subjects kept a 3-d weighed food intake record which was used to estimate habitual dietary intakes using the Compeat 4 program (Nutrition Systems, London). The subjects reported no alcohol intake during the food recording period and habitually only occasionally consumed alcohol. Details of the subjects are given in Table 1.

\section{Experimental design}

A randomized crossover design was used to compare the effects of six different test meals which consisted of a muffin and milk-shake. Five high-fat $(50 \mathrm{~g})$ meals were compared with a low-fat $(15 \mathrm{~g})$ test meal. Each subject consumed the six test meals with at least 1 week between treatments. The subjects were recruited in three groups of six subjects and the order of allocation of the test meals was

Table 1. Characteristics of the female subjects ( $n$ 18) (Mean values and standard deviations)

\begin{tabular}{lrr}
\hline & Mean & SD \\
\hline Age (years) & 28.2 & 6.4 \\
BMI (kg/m²) & 21.4 & $1 \cdot 7$ \\
Energy intake (MJ/d) & 7.6 & 1.2 \\
Protein intake (\% energy) & 15.0 & 1.2 \\
Fat intake (\% energy) & 39.1 & 4.2 \\
Carbohydrate intake (\% energy) & 46.5 & $5 \cdot 1$ \\
Fibre intake (g/d) & 11.8 & 2.7 \\
Plasma triacylglycerol (mmol/l) & 0.9 & 0.3 \\
Plasma cholesterol (mmol/l) & 4.5 & 2.4 \\
\hline
\end{tabular}

done according to an orthogonal Latin square design. The test meal consisted of a muffin and a milk-shake and was devised so that $30 \mathrm{~g}$ of the fat was held constant (about $20 \mathrm{~g}$ $18: 1 n-9,6 \mathrm{~g} 18: 2 n-6,2 \mathrm{~g} 16: 0,2 \mathrm{~g} 18: 0)$ and $20 \mathrm{~g}$ consisted of the test fatty acids. The oils used in the present study were a high-oleic sunflower oil, palm oil (rich in palmitic acid), a blend of high-oleic sunflower oil and MCT, safflower oil (rich in linoleic acid), and a blend of higholeate sunflower oil and fish-oil concentrate, which was devoid of cholesterol. The low-fat meal contained $15 \mathrm{~g}$ higholeate sunflower oil and the energy content of the meal was maintained constant, increasing the carbohydrate content of the meal by using polydextrose solution (Polycal; Cow and Gate, Trowbridge, Wilts., UK). The fatty acid composition of the oils was determined by GLC and the nutrient composition calculated from food tables. The nutrient content of the meals is shown in Table 2.

Fasting venous blood samples were collected using the vacutainer technique into ETDA-containing vacutainers following an overnight fast. The subjects consumed the test meal within $30 \mathrm{~min}$ and further venous blood samples were collected 30, 60 and $180 \mathrm{~min}$ from the beginning of the meal. Plasma was separated by centrifugation at $1500 \mathrm{~g}$ for $15 \mathrm{~min}$ at $4^{\circ} \mathrm{C}$ and stored at $-20^{\circ} \mathrm{C}$ until analysed for plasma bile acids and CCK. The protocol was approved by the Research Ethics Committee of King's College London and the subjects gave written informed consent.

\section{Laboratory methods}

Plasma bile acids were analysed by GC-MS (Clayton \& Muller, 1980). Briefly, an internal standard of nordeoxycholic acid (Catalogue no. N2000; Steraloids, Newport, RI, USA) was added to the plasma sample and bile acids were deconjugated with choline glycine hydrolase (SigmaAldrich catalogue C4018; Poole, Dorset, UK) and extracted with alkaline XAD-2 resin. The bile acids were recovered by eluting sequentially with $2 \mathrm{ml}$ of a mixture of hexanechloroform-methanol (1:1:1, by vol.) and twice with $2 \mathrm{ml}$ methanol. The bile acids were methylated with diazomethane and trimethyl silylated with Tri-Sil (Catalogue no. 48999, Pierce, Rockford, IL 611101, USA) and analysed on a Hewlett Packard 6890 mass spectrometer using a $25 \mathrm{~m}$ BPX35, $0.22 \mathrm{~mm}$ internal diameter and $0.25 \mu \mathrm{m}$ film thickness (SGE Europe, Milton Keynes MK11 3LA, UK). The initial column temperature was $80^{\circ} \mathrm{C}$, which was held for $2 \mathrm{~min}$ and then increased at $100^{\circ} \mathrm{C} / \mathrm{min}$ to $200^{\circ} \mathrm{C}$ for $4 \mathrm{~min}$ then increased at $2^{\circ} \mathrm{C} / \mathrm{min}$ to $300^{\circ} \mathrm{C}$ and held at that temperature for $4 \mathrm{~min}$. The injector temperature and transfer line temperatures were set at $250^{\circ} \mathrm{C}$. Bile acids were identified by their mass spectra and quantified in relation to internal standard.

Plasma CCK was analysed by radioimmunoassay (Jordinson et al. 1998). The CCK was extracted from $1 \mathrm{ml}$ plasma with $2 \mathrm{ml}$ ethanol and the dried extract reconstituted into assay buffer. Labelled CCK-8 (sulfated $\mathrm{I}^{125}$, catalogue no. D41349; Amersham International, Bucks., UK) and antiserum to CCK was raised by immunizing a rabbit with CCK-10 conjugated to keyhole limpet haemocyanin, using carbodimide. This antiserum was specific for both CCK-8 and CCK-33, which constitute the majority of the CCK 
Table 2. Nutrient composition of the test meals*

\begin{tabular}{|c|c|c|c|c|c|c|}
\hline Test meal.. & Low-fat & MCT & Palmitate & Oleate & $n$-3+Oleate & Linoleate \\
\hline Energy (MJ) & $4 \cdot 8$ & $4 \cdot 8$ & $4 \cdot 8$ & $4 \cdot 8$ & $4 \cdot 8$ & $4 \cdot 8$ \\
\hline Protein (\% energy) & 12 & 12 & 12 & 12 & 12 & 12 \\
\hline Carbohydrate (\% energy) & 76 & 48 & 48 & 48 & 48 & 48 \\
\hline Fat (\% energy) & 12 & 40 & 40 & 40 & 40 & 40 \\
\hline Fibre (g) & 4 & 4 & 4 & 4 & 4 & 4 \\
\hline Fat $(\mathrm{g})$ & 15 & 50 & 50 & 50 & 50 & 50 \\
\hline Capric acid (8:0) (g) & 0 & $10 \cdot 6+$ & 0 & 0 & 0 & 0 \\
\hline Caproic acid (10:0) (g) & 0 & $10 \cdot 3 \dagger$ & 0 & 0 & 0 & 0 \\
\hline Palmitic acid $(16: 0)(\mathrm{g})$ & 0.6 & 1.2 & $20 \cdot 5+$ & $2 \cdot 1$ & 1.9 & $2 \cdot 6$ \\
\hline Stearic acid $(18: 0)(\mathrm{g})$ & 0.6 & $1 \cdot 1$ & $2 \cdot 1$ & $2 \cdot 1$ & 1.8 & 1.7 \\
\hline Oleic acid $(18: 1 n-9)(\mathrm{g})$ & $11 \cdot 4$ & $19 \cdot 1$ & $19 \cdot 1$ & $38 \cdot 2 \dagger$ & $32 \cdot 6 \dagger$ & $23 \cdot 6$ \\
\hline Linoleic acid $(18: 2 n-6)(\mathrm{g})$ & 1.4 & $4 \cdot 8$ & $4 \cdot 8$ & $4 \cdot 6$ & 3.9 & $19 \cdot 1 \dagger$ \\
\hline EPA $(20: 5 n-3)(g)$ & 0 & 0 & 0 & 0 & $3.4 \dagger$ & 0 \\
\hline DPA $(22: 5 n-3)(g)$ & 0 & 0 & 0 & 0 & $0.5 \dagger$ & 0 \\
\hline DHA $(22: 6 n-3)(g)$ & 0 & 0 & 0 & 0 & $1.4 \dagger$ & 0 \\
\hline Saturated fatty acids (g) & 1.6 & 23.9 & 24 & $5 \cdot 1$ & 5 & $5 \cdot 1$ \\
\hline Monounsaturated fatty acids (g) & 11.4 & $19 \cdot 1$ & $19 \cdot 1$ & $38 \cdot 2$ & $33 \cdot 6$ & $23 \cdot 6$ \\
\hline Polyunsaturated fatty acids (g) & 1.4 & 4.8 & $4 \cdot 8$ & 4.6 & $9 \cdot 2$ & $19 \cdot 1$ \\
\hline
\end{tabular}

MCT, medium-chain triacylglycerol; EPA, eicosapentaenoic acid; DPA, docosapentaenoic acid; DHA, docosahexaenoic acid. ${ }^{*}$ For details of subjects and procedures, see Table 1 and pp. 472-473.

†Fat exchanges between the high-fat test meals.

present in plasma. The lower detection limit was $0 \cdot 2 \mathrm{pmol} / \mathrm{l}$. The inter- and intra-assay variability were 12 and $6 \cdot 2 \%$ respectively.

\section{Statistical analysis}

Data were analysed by repeated measures ANOVA using SPSS/PC version 8.0. Where there was a significant difference between fasting and non-fasting samples, the deviations from fasting were calculated and separately analysed. Newman-Keuls multiple comparison test was used to test for differences between treatments using GraphPad Prism software version 3.01 (GraphPad Inc, California CA92121, USA). The incremental area under the curve (AUC) was estimated using GraphPad Prism.

\section{Results}

Two subjects did not complete the study, one consumed five of the six meals and the other consumed four of the six meals. The meals were generally well tolerated by the subjects except the MCT meal, which caused nausea and gastrointestinal discomfort in two subjects. Table 3 shows the plasma CCK concentrations before and after the six test meals. Plasma CCK increased following all test meals (time effect $P<0 \cdot 0001$ ). The postprandial increase in CCK from fasting at $30 \mathrm{~min}$ was significantly lower after the MCT meal compared with all the other meals and the increase from fasting was greater after the linoleate meal compared with the low-fat, palmitate and $n$-3+oleate meals (Table 3 ). At 60 min the postprandial increase was significantly lower after the MCT meal compared with the linoleate meal. The integrated AUC (Fig. 1) for plasma CCK showed significant differences between treatments $(P=0 \cdot 0001)$. Values were significantly lower following the MCT meal compared with the other high-fat meals $(P<0 \cdot 05)$.

Fasting plasma total bile acid values did not differ before the meals (Table 4). Plasma total bile acid increased significantly

Table 3. Fasting and postprandial plasma cholecystokinin concentrations (pmol/l) following six different test meals in sixteen healthy premenopausal women*

(Mean values with their standard errors)

\begin{tabular}{|c|c|c|c|c|c|c|c|c|}
\hline \multirow[b]{2}{*}{ Test meal } & \multicolumn{2}{|c|}{ Fasting } & \multicolumn{2}{|c|}{30 mint } & \multicolumn{2}{|c|}{60 mint } & \multicolumn{2}{|c|}{$180 \min \dagger$} \\
\hline & Mean & SEM & Mean & SED & Mean & SED & Mean & SED \\
\hline Low-fat & $2 \cdot 0$ & 0.39 & $6 \cdot 0^{\mathrm{a}}$ & 0.87 & $6 \cdot 1^{\mathrm{a}}$ & 0.70 & $5 \cdot 8$ & 0.56 \\
\hline MCT & $1 \cdot 8$ & 0.32 & $3 \cdot 4^{\mathrm{b}}$ & 0.36 & $4 \cdot 4^{\mathrm{b}}$ & 0.50 & $4 \cdot 3$ & 0.48 \\
\hline Palmitate & 1.6 & 0.27 & $5 \cdot 8^{\mathrm{a}}$ & 0.75 & $5 \cdot 9^{\mathrm{ab}}$ & 0.81 & $5 \cdot 7$ & 0.80 \\
\hline Oleate & $1 \cdot 7$ & 0.31 & $7 \cdot 0^{\mathrm{ac}}$ & $1 \cdot 10$ & $8 \cdot 1^{\mathrm{ab}}$ & 1.30 & $5 \cdot 1$ & 0.84 \\
\hline$n$-3+Oleate & $2 \cdot 0$ & 0.29 & $5 \cdot 9^{\mathrm{a}}$ & 0.78 & $6 \cdot 8^{\mathrm{ab}}$ & 0.68 & $6 \cdot 0$ & 1.09 \\
\hline Linoleate & 1.8 & 0.34 & $8 \cdot 4^{\mathrm{C}}$ & 1.09 & $8 \cdot 4^{\mathrm{a}}$ & 1.35 & 8.0 & 1.07 \\
\hline Significance of $F$ & \multicolumn{2}{|c|}{ NS } & \multicolumn{2}{|c|}{$P<0.0001$} & \multicolumn{2}{|c|}{$P=0.0137$} & \multicolumn{2}{|c|}{ NS } \\
\hline
\end{tabular}

MCT, medium-chain triacylglycerol.

${ }^{\mathrm{a}, \mathrm{b}, \mathrm{c}}$ Mean values within a column with unlike superscript letters were significantly different, $P<0.05$; Newman-Keuls multiple comparison test.

${ }^{*}$ For details of subjects and procedures, see Tables 1 and 2 and pp. 472-473.

†Non-fasting values are all significantly different from the fasting value in the same row, $P<0.01$. 


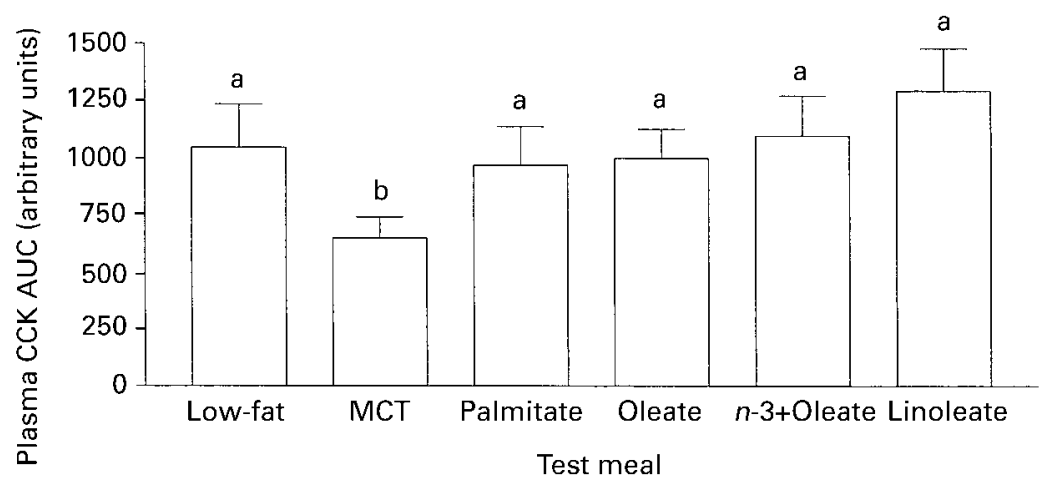

Fig. 1. Mean incremental area under the curve (AUC) for plasma cholecystokinin (CCK) concentration following six different test meals. Standard errors of the mean values are represented by vertical bars. Bars with unlike superscript letters were significantly different $(P<0 \cdot 05)$. MCT, medium-chain triacylglycerol.

from fasting following all test meals $(P<0 \cdot 001)$ and remained elevated up to $3 \mathrm{~h}$ after the meal. There were significant differences between meals at 30,60 and 180 min postprandially. The postprandial increase in total bile acids was significantly lower after the MCT meal compared with the oleate $+n-3$ and linoleate meals at $30 \mathrm{~min}$. The increase in total bile acid at $60 \mathrm{~min}$ was significantly lower after the MCT meal compared with the linoleate meal, oleate and oleate $+n-3$ meals; it was also significantly lower after the low-fat compared with the linoleate meal. At $180 \mathrm{~min}$ the postprandial increase in total bile acids was significantly lower after the MCT meal compared with the linoleate and oleate $+n-3$ meals and was significantly lower after the low-fat meal compared with the linoleate meal. The AUC was calculated and found to differ significantly between meals $(P<0 \cdot 0001)$. The AUC (Fig. 2) was significantly lower following the MCT meals compared with oleate, oleate $+n-3$ and linoleate meals $(P<0.01, P<0.001$ and $P<0.001$ respectively) and was greater following the linoleate meal compared with the low-fat and palmitate meal (both $P<0 \cdot 01$ ). CDCA, DCA and CA were the major bile acids detected in plasma. However, in one subject DCA was not detectable. Smaller amounts of lithocholic acid and ursodeoxycholic acid were detected in some but not all subjects; owing to the low concentrations present these results are not reported. Fig. 3 shows the AUC for the major plasma bile acids; the main increase was in the concentration of CDCA. The AUC for plasma CDCA was greater following the oleate, oleate $+n-3$ and linoleate meals than following both the low-fat and MCT meals. The AUC for CDCA was also greater following the linoleate meal compared with the palmitate meal $(P<0 \cdot 01)$. The postprandial AUC was significantly greater for $\mathrm{CA}$ following the linoleate and the oleate meals compared with the MCT meal. The postprandial AUC for DCA was significantly greater following the linoleate and $n$-3+oleate meals compared with the MCT meal $(P<0 \cdot 05)$. Neither the DCA:CA or CA:DCA changed significantly postprandially or between meals.

\section{Discussion}

The purpose of the present study was to test the hypothesis that fatty acids differ according to chain length and unsaturation with regard to their effects on CCK release and the postprandial increase in bile acid concentrations. In order to achieve this objective subjects were fed meals where about two-fifths of the fatty acids were substituted

Table 4. Fasting and postprandial plasma total bile acid concentrations ( $\mu \mathrm{mol} / \mathrm{l})$ following six different test meals in sixteen healthy premenopausal women*

(Mean values with their standard errors)

\begin{tabular}{|c|c|c|c|c|c|c|c|c|}
\hline \multirow[b]{2}{*}{ Test meal } & \multicolumn{2}{|c|}{ Fasting } & \multicolumn{2}{|c|}{$30 \min \uparrow$} & \multicolumn{2}{|c|}{$60 \min \uparrow$} & \multicolumn{2}{|c|}{180 mint } \\
\hline & Mean & $\overline{\text { SEM }}$ & Mean & $\overline{\text { SED }}$ & Mean & $\overline{\mathrm{SED}}$ & Mean & SED \\
\hline Low-fat & 2 & 0.38 & $4 \cdot 7^{\mathrm{ab}}$ & 0.37 & $3 \cdot 8^{a b}$ & 0.38 & $3 \cdot 1^{\mathrm{ab}}$ & $0 \cdot 41$ \\
\hline MCT & 1.9 & 0.25 & $2 \cdot 8^{a}$ & 0.34 & $2 \cdot 6^{\mathrm{b}}$ & 0.32 & $2 \cdot 5^{\mathrm{b}}$ & 0.39 \\
\hline Palmitate & $1 \cdot 7$ & 0.22 & $4 \cdot 1^{\mathrm{ab}}$ & 0.39 & $3 \cdot 7^{\mathrm{ab}}$ & 0.42 & $3 \cdot 8^{\mathrm{abc}}$ & 0.47 \\
\hline Oleate & 1.8 & 0.4 & $4 \cdot 7^{\mathrm{ab}}$ & 0.77 & $5 \cdot 4^{\mathrm{ac}}$ & 0.83 & $3 \cdot 6^{\mathrm{abc}}$ & 0.5 \\
\hline$n$-3+Oleate & $2 \cdot 1$ & 0.37 & $5 \cdot 9^{\mathrm{b}}$ & 0.91 & $5 \cdot 5^{\mathrm{ac}}$ & 0.98 & $5 \cdot 2^{\mathrm{ac}}$ & 0.6 \\
\hline Linoleate & 2 & 0.3 & $6 \cdot 9^{b}$ & 1.02 & $6 \cdot 5^{\mathrm{c}}$ & 1.23 & $5 \cdot 3^{c}$ & 0.85 \\
\hline Significance of $F$ & \multicolumn{2}{|c|}{ NS } & \multicolumn{2}{|c|}{$P=0.0021$} & \multicolumn{2}{|c|}{$P=0.0016$} & \multicolumn{2}{|c|}{$P=0.003$} \\
\hline
\end{tabular}

MCT, medium-chain triacylglycerol.

a,b,c Mean values within a column with unlike superscript letters were significantly different, $P<0.05$; NewmanKeuls multiple comparison test.

* For details of subjects and procedures, see Tables 1 and 2 and pp. 472-473.

$\dagger$ Non-fasting values are all significantly different from the fasting value in the same row, $P<0.01$. 


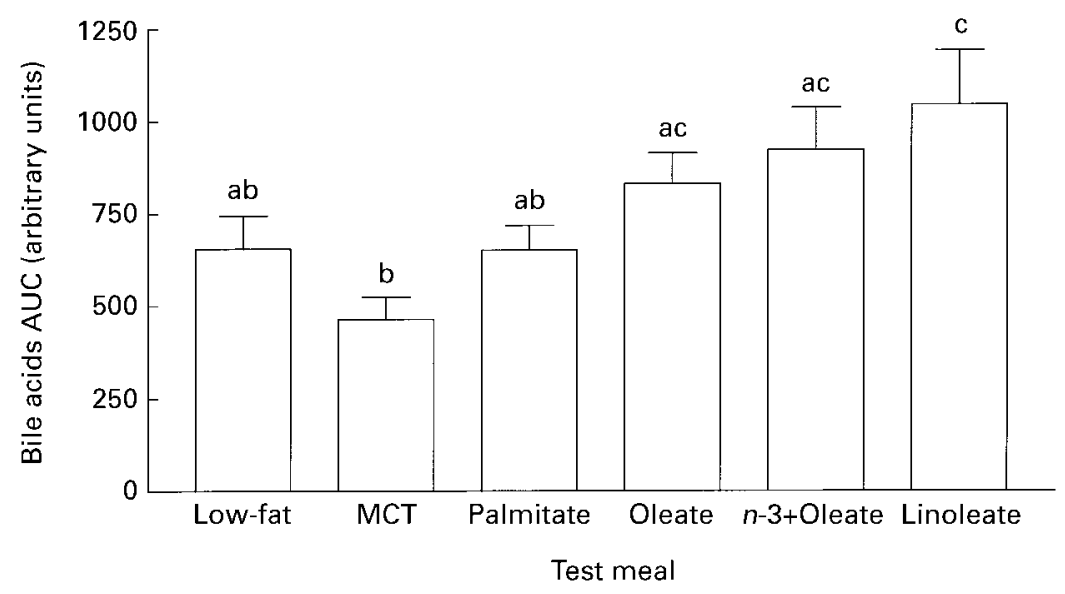

Fig. 2. Mean incremental area under the curve (AUC) for plasma total bile acid concentrations following six different test meals. Standard errors of the mean values are represented by vertical bars. Bars with unlike superscript letters were significantly different $(P<0.05)$. MCT, medium-chain triacylglycerol.

with the test fatty acids and comparisons were made with a low-fat test meal. The low-fat test meal resulted in a similar elevation of CCK and bile acids compared with some of the high-fat meals. Both plasma CCK and bile acid concentrations did not differ significantly following the low-fat meal compared with the high-oleate meal, which were both constituted using high-oleic sunflower oil (50 g in the higholeate meal and $15 \mathrm{~g}$ in the low-fat meal). This finding was unexpected and suggests that the amount of fat in a meal is not a major determinant of CCK release.

There was a rapid increase in plasma CCK concentration 30 min following the beginning of the test meal. The results generally support the finding of Beardshall et al. (1989), who suggested that CCK release increased with degree of unsaturation. However, the initial increase following the linoleate meal was greater than that following the $n$-3+oleate meal; the unsaturation index was 0.62 in the linoleate meal compared with 0.68 in the $n$-3+oleate meal. This observation is consistent with other data that suggest that $n-6$ and $n-3$ fatty acids may have differing effects on CCK release. Jonkers et al. (2000) investigated the effects of intraduodenally administered fish oil (rich in $n-3$ fatty acids) compared with maize oil (rich in $n-6$ fatty acids) on CCK release and gall bladder contraction in nine healthy volunteers and reported that gall bladder contraction duration was significantly shorter after fish oil than maize oil and CCK secretion was reduced. The linoleate meal resulted in a sustained increase in plasma bile acid concentrations, particularly that of chenodeoxycholic acid, compared with the low-fat, MCT and palmitate meals. High intakes of linoleic acid are known to have a cholesterol-lowering effect and this may be mediated by an increased secretion of bile (Becker et al. 1983). However, it is also of interest to note that Sturdevant et al. (1973) reported a higher incidence of gallstones in subjects receiving a diet enriched in linoleic acid compared with a diet rich in saturated fatty acids. The findings of the present study suggest that meals rich in linoleic acid lead to greater and more sustained elevation of plasma bile acid concentrations. A limitation of the present study is that it

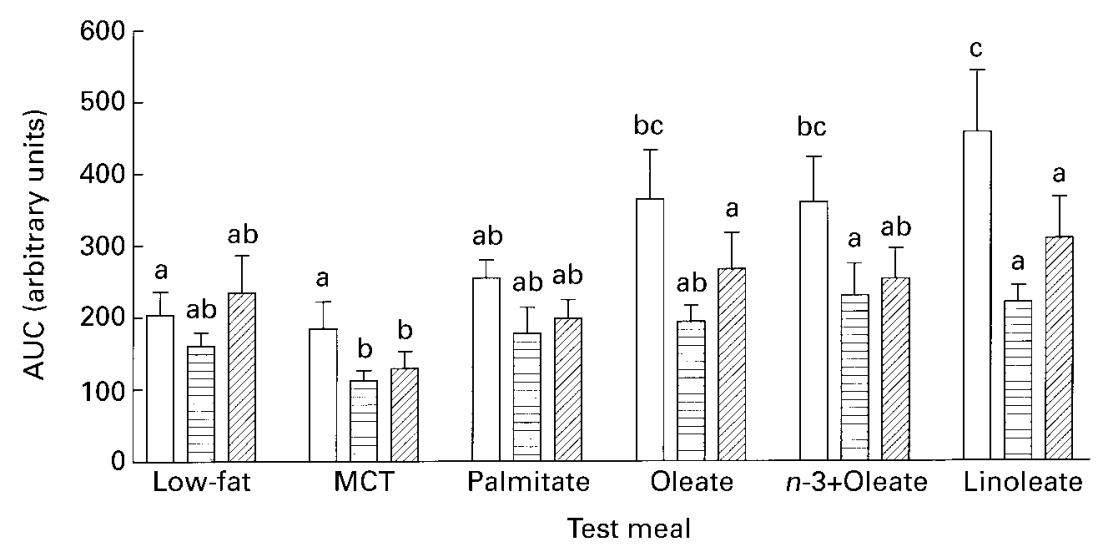

Fig. 3. Mean incremental area under the curve (AUC) for plasma cholic (目), chenodeoxycholic ( $\square$ ) and deoxycholic (四) acid concentrations following six different test meals. Standard errors of the mean values are represented by vertical bars. Bars with unlike superscript letters were significantly different $(P<0.05)$. MCT, medium-chain triacylglycerol. 
relates to the effects of a single meal rather than habitual intake and it is possible that adaptation may occur in the long term. The results from our study might be taken to imply that meals rich in linoleic acid may increase risk of breast cancer especially if consumed frequently. However, a large prospective cohort study found no consistent relationship between either the proportion of energy from fat or polyunsaturated fatty acids and risk of breast cancer (Holmes et al. 1999).

It has been proposed that the bacterial degradation of primary into secondary bile acids may be associated with increased risk of colorectal and breast cancer (Lewis \& Heaton, 1999). It has previously been shown that DCA:CA is influenced by diet (Reddy et al. 1998) and particularly by the availability of fermentable carbohydrate which results in decreased colonic $\mathrm{pH}$, which inhibits the bacterial degradation of primary bile acids. The present study demonstrates that significant concentrations of DCA can be detected in blood plasma and that DCA:CA did not change postprandially. This would suggest that DCA:CA in plasma could be used as an accessible index of the bile acid degradation in epidemiological studies.

The most striking observation was that the MCT meal suppressed the rise in CCK and plasma bile acid compared with all the other meals. Isaacs et al. (1987) reported that the gall bladder in human subjects does not contract after the ingestion of MCT. McLaughlin et al. (1999) infused individual fatty acids into the upper gut in healthy volunteers and reported that plasma CCK concentrations were elevated by fatty acids with a chain of twelve $\mathrm{C}$ atoms or longer, whereas those of eleven or fewer $\mathrm{C}$ atoms failed to increase plasma CCK. In the present study, the MCT was administered as part of a test meal that contained a significant amount of long-chain fatty acids (indeed more than in the low-fat meal) yet the increase in plasma CCK was lower in comparison with the low-fat meal. The inhibitory effect of MCT on plasma CCK is in contrast to the effects in rats and chickens (Douglas et al. 1990; Mabayo et al. 1992). MCT differ from long-chain triacylglycerols in that they are more rapidly hydrolysed and are transported in the hepatic portal vein to the liver. The location of the CCK-producing cells is in the intestinal mucosa, with its apical surface exposed to the lumen (Liddle, 1997), might be affected by luminal fatty acid concentrations. Receptors responsive to fatty acids have been identified in the gastrointestinal tract. Inhibition of long-chain triacylglycerol digestion has been shown to cause a reduction in plasma CCK concentration indicating that free fatty acids are an important stimulus for CCK secretion (Feinle et al. 2001). However, addition of a lipase inhibitor to MCT did not modify the inhibitory effect of MCT on CCK secretion. Peptide PYY is known to suppress the production of CCK. Jakob et al. (2000) reported that infusion of MCT, as opposed to a triacylglycerol consisting mainly of stearic and palmitic acid in pigs, led to a rapid decrease in plasma CCK, which was accompanied by a decrease in pancreatic juice secretion decreasing the output of colipase and lipase; however, plasma PYY secretion was not influenced by MCT. Further studies are required to determine the mechanism of action by which MCT inhibits CCK secretion.

\section{References}

Baker PR, Wilton JC, Jones CE, Stenzel DJ, Watson N \& Smith GJ (1992) Bile acids influence the growth, oestrogen receptor and oestrogen-regulated proteins of MCF-7 human breast cancer cells. British Journal of Cancer 65, 566-572.

Bayerdorffer E, Mannes GA, Ochsenkuhn T, Dirschedl P, Wiebecke B \& Paumgartner G (1995) Unconjugated secondary bile acids in the serum of patients with colorectal adenomas. Gut 36, 268-273.

Beardshall K, Frost G, Morarji Y, Domin J, Bloom SR \& Calam J (1989) Saturation of fat and cholecystokinin release: implications for pancreatic carcinogenesis. Lancet ii, 1008-1010.

Becker N, Illingworth DR, Alaupovic P, Connor WE \& Sundberg EE (1983) Effects of saturated, monounsaturated, and omega-6 polyunsaturated fatty acids on plasma lipids, lipoproteins, and apoproteins in humans. American Journal of Clinical Nutrition 37, 355-360.

Clayton PT \& Muller DP (1980) A simplified gas-liquid chromatographic method for the estimation of non-sulphated plasma bile acids. Clinica Chimica Acta 105, 401-405.

Douglas BR, Jansen JB, de Jong AJ \& Lamers CB (1990) Effect of various triglycerides on plasma cholecystokinin levels in rats. Journal of Nutrition 120, 686-690.

Feinle C, Rades T, Otto B \& Fried M (2001) Fat digestion modulates gastrointestinal sensations induced by gastric distention and duodenal lipid in humans. Gastroenterology 120, 1100-1107.

Hill MJ, Goddard P \& Williams RE (1971) Gut bacteria and aetiology of cancer of the breast. Lancet ii, 472-473.

Holmes MD, Hunter DJ, Colditz GA, Stampfer MJ, Hankinson SE, Speizer FE, Rosner B \& Willett WC (1999) Association of dietary intake of fat and fatty acids with risk of breast cancer. Journal of the American Medical Association 281, 914-920.

Isaacs PE, Ladas S, Forgacs IC, Dowling RH, Ellam SV, Adrian TE \& Bloom SR (1987) Comparison of effects of ingested medium- and long-chain triglyceride on gallbladder volume and release of cholecystokinin and other gut peptides. Digestive Diseases Science 32, 481-486.

Jakob S, Mosenthin R, Zabielski R, Rippe C, Winzell MS, Gacsalyi U, Laubitz D, Grzesiuk E \& Pierzynowski SG (2000) Fats infused intraduodenally affect the postprandial secretion of the exocrine pancreas and the plasma concentration of cholecystokinin but not of peptide YY in growing pigs. Journal of Nutrition 130, 2450-2455.

Javitt NB, Budai K, Miller DG, Cahan AC, Raju U \& Levitz M (1994) Breast-gut connection: origin of chenodeoxycholic acid in breast cyst fluid. Lancet 343, 633-635.

Jonkers IJ, Ledeboer M, Steens J, Smelt AH \& Masclee AA (2000) Effects of very long chain versus long chain triglycerides on gastrointestinal motility and hormone release in humans. Digestive Diseases Science 45, 1719-1726.

Jordinson M, Beales IL \& Calam J (1998) Soybean agglutinin stimulated cholecystokinin release from cultured rabbit jejunal cells requires calcium influx via L-type calcium channels. Peptides 19, 1541-1547.

Kawasumi H, Kaibara N \& Koga S (1988) Cocarcinogenic activity of bile acids in the chemical transformation of $\mathrm{C} 3 \mathrm{H} / 10 \mathrm{~T} 1 / 2$ fibroblasts in vitro. Oncology 45, 192-196.

Kucio C, Besser P \& Jonderko K (1988) Gallbladder motor function in obese versus lean females. European Journal of Clinical Nutrition 42, 121-124.

Lewis SJ \& Heaton KW (1999) The metabolic consequences of slow colonic transit. American Journal of Gastroenterology $\mathbf{9 4 ,}$ 2010-2016. 
Liddle (1997) Cholecystokinin cells. Annual Reviews in Physiology 59, 735-756.

Mabayo RT, Furuse M, Yang SI \& Okumura J (1992) Mediumchain triacylglycerols enhance release of cholecystokinin in chicks. Journal of Nutrition 122, 1702-1705.

McLaughlin J, Grazia LM, Jones MN, D'Amato M, Dockray GJ \& Thompson DG (1999) Fatty acid chain length determines cholecystokinin secretion and effect on human gastric motility. Gastroenterology 116, 46-53.

Mössner J, Grumann M, Zeeh J \& Fischbach W (1992) Influence of various nutrients and their mode of application on plasma cholecystokinin (CCK) bioactivity. Clinical Investigation 70, $125-129$.
Raju U, Levitz M \& Javitt NB (1990) Bile acids in human breast cyst fluid: the identification of lithocholic acid. Journal of Clinical Endocrinology and Metabolism 70, 1030-1034.

Reddy S, Sanders TA, Owen RW \& Thompson MH (1998) Faecal $\mathrm{pH}$, bile acid and sterol concentrations in premenopausal Indian and white vegetarians compared with white omnivores. British Journal of Nutrition 79, 495-500.

Sturdevant RA, Pearce ML \& Dayton S (1973) Increased prevalence of cholelithiasis in men ingesting a serumcholesterol-lowering diet. New England Journal of Medicine 288, 24-27.

Watabe J \& Bernstein H (1985) The mutagenicity of bile acids using a fluctuation test. Mutation Research 158, 45-51. 